\title{
Performance Comparison for Omnidirectional and Directional MAC Protocols for Ad hoc Network
}

\author{
Asrar Ahmad Ansari \\ College of e-Learning \& \\ Distance Learning \\ Deanship \\ King Saud University, \\ Riyadh
}

Saudi Arabia- 11461

\author{
Zafrul Hasan \\ E- Learning and IT Unit \\ College of Nursing \\ King Saud University, \\ Riyadh \\ Saudi Arabia- 11485
}

\author{
Md. Jahangeer Alam \\ Department of Computer \\ and Information Science \\ Jazan Community College \\ Jazan University, Jazan
}

\author{
Khwaja Mohammad \\ Athar Siddique \\ College of Computer \\ Science \\ Jazan University \\ Jazan, Saudi Arabia
}

\begin{abstract}
In ad hoc networks, traditional MAC protocols used omnidirectional antennas. But these days directional antennas have emerged as an alternative of omnidirectional antenna. The directional antennas offer many benefits compared to classical omnidirectional antennas. Those include significant increases in spatial reuse, coverage range and subsequently network capacity as a whole. In this paper, we present comparison study of directional and omnidirectional MAC protocols. We are contrasting their features and evaluating their performance under various network load. For comparison the performance we simulated some omnidirectional and directional MAC protocols by using OPNET 14.5. we have simulated an ad hoc network scenarios by using the omnidirectional and directional MAC protocols
\end{abstract}

\section{Keywords}

WLAN, Ad hoc Networks, IEEE 802.11, directional MAC, hidden terminal problem, exposed node problem.

\section{INTRODUCTION}

Wireless ad hoc networks are dynamically formed amongst a group of wireless users and require no existing infrastructure or preconfiguration. When Carrier Sense Medium Access (CSMA) protocol applied to ad hoc networks, causes the hidden terminal and the exposed terminal problems [I]. After that to overcome these problem, Medium Access Collision Avoidance (MACA) [2] proposed. To overcome these problem MACA proposed RTS and CTS (Request-To-Send and Clear-To-Send) frames. Further the authors improved MACA and proposed MACAW [3] with more optional control frames. Non-persistent carrier sensing and the RTSiCTS scheme together proposed in Floor Acquisition Multiple Access (FAMA) [4]. , the IEEE 802.11 [5] MAC protocol is the most widely used protocol. It is based on the concept of Carrier Sense Multiple Access with Collision Avoidance (CSMA/CA).

The above discussed MAC protocols are usages omnidirectional antenna. With the omnidirectional antenna there is very few chance to use spatial reuse. The unwanted interference also happens from other direction with omnidirectional antennas. The directional antenna technology provides plenty of opportunities for communication networks to achieve healthier performance output on all the traditional parameters like delay, throughput, energy consumption, etc. The directional antenna based MAC protocols are capable to transmit packets in a certain directions. The directional transmission reduces the chance of collision and increase the effective network capacity. There are several directional MAC proposed for ad hoc networks. YoungBae Ko et-al,[6] proposed a scheme in which the all the fundamental of IEEE 802.11 MAC like RTS/CTS handshake, network allocation vector, channels sensing and backoff have been incorporated. The only difference is that transmission and reception of the packet was directional instead of omnidirectional. They succeed to achieve better network throughput. However, it has to need extra location tracking support. Nasipuri et-al [7], proposed scheme overcomes the above problem. In his scheme the RTS/CTS frames transmits omnidirectional to identify the direction of communicating node with the help of Angle Of Arrival (AOA). The data and Ack transmits directionally. However, it didn't have the benefit of reserving the channel directionally. Gossain et al. [8] proposed scheme decreases the shortcoming of previous MAC by sending the optimized circular RTS/CTS after the successful RTS/CTS handshake. But due to omnidirectional reception, the hidden node problem arises asymmetry in gain caused hidden node problem. Alam et-al [9] proposed a scheme overcomes this problem. In his scheme the all transmission is directional. They uses multibeam smart antenna, which cover the whole area around the node.

In this paper, we comparison the performance of omnidirectional and directional MAC protocols via simulation. For simulation we uses OPNET 14.5 [10]. We design a ad hoc network scenario and runs it for each directional and omnidirectional MAC protocols.

The rest of the paper is organized as follows. Section 2 includes the motivation of our work. Section 3 contains brief review of directional and omnidirectional MAC protocols. In section 4 we compares the features of MAC protocols. Section 5 includes simulation model and results, and Section 6 concludes the work.

\section{MOTIVATION}

Our work is motivated by [11], in that paper they compares the omnidirectional and directional MAC protocols for Ad-hoc network. We also compare the basic omni directional MAC with directional MAC. But our paper includes recent directional MAC protocols like DMAC3 and DMAC4. We simulate the protocols on opnet 14.5 and compare the results in throughput and delay metric.

\section{SUMMARY OF MAC PROTOCOLS}

\section{A. Omnidirectional MAC Protocols}

Following are the brief overview of Omnidirectional MAC protocols.

\section{i. IEEE 802.11 DCF}

The IEEE 802.11 [5] MAC protocol is the most widely used protocol In wireless ad hoc networks. It is based on the concept of Carrier Sense Multiple Access with Collision Avoidance (CSMA/CA). In this protocol, when the frame size exceeds certain threshold, the RTS/CTS scheme is used in addition to carrier sensing. When a node hears an RTS or a CTS frame, it will set the 
NAV (Network Allocation Vector) to defer itself from access until the end of the corresponding data transmission to avoid collision with the ACK frame. When the virtual sensing is used, the hidden terminal problem is relieved while the exposed terminal problem emerges; otherwise the hidden terminal problem exists, while the exposed terminal problem is not significant.To resolve channel contention the IEEE 802.11 MAC protocol uses a back off mechanism in which the node chooses a random back off interval from $[0, \mathrm{CW}]$, if the channel is found to be busy during the physical carrier sensing. The node decrements the back off counter by one for every idle slot time increment, and when it reaches zero, the node then transmits its packet.

ii.

\section{$M A C A$ and $M A C A W$}

MACA uses two types of short, fixed-size signaling packets. When station A wishes to transit packet to station $\mathrm{B}$, it sends a RTS packet to B. If station B hears the RTS, and it is not currenetly deferring, it immediately replies with a CTS packet. Upon receiving the CTS, station A immediately sends its data. The neighbouring stations who overhearing an RTS defer all transmissions until some time after the associated CTS packet would have finished. The station who overhearing a CTS packet defers for the length of the expected data transmission.

MACA uses only the virtual sensing. The subtle difference between MACA's RTS/CTS scheme and that of the IEEE 802.11 MAC DCF is that, when a node overhears an RTS frame, instead of deferring until the end of the data transmission, it only defers until the corresponding CTS frame is expected to be received. The consequence is that the exposed terminal problem, in addition to the hidden terminal problem, is also solved. MACAW basically follows the same RTS/CTS scheme as MACA, while it introduces more optional control frames and a different backoff algorithm.

iii.

Floor Acquisition Multiple Access (FAMA)

Like IEEE 802.11 MAC, FAMA exploits both carrier sensing and virtual sensing. It combines non-persistent carrier sensing with the virtual sensing scheme of MACA, which is referred to as FAMANTR (FAMA Non-persistent Transmit Request). When a station has one or multiple packets to deliver, it first listens to the channel. If the channel is busy, the station backs off and tries to re- transmit at a later time using a random value for the backoff time. If the channel is clear, the station transmits a RTS. The sender listens to the channel from one round-trip time plus the time needed for the destination to send a CTS. If the CTS packet is corrupted or is not received within the time limit, the sender goes into the back-off state and tries to retransmit at a later time. When the originator receives the CTS from the destination, it begins its transmission of the data packet burstt. The burst is limited to a maximum number of data packets, after which the station must release the channel and contend to re-acquire the floor. This variant of FAMA is similar to the protocol proposed for IEEE 802.11[5], and Apple's Local Talk Link Access protocol [12].

\section{B. Directional Antenna Model}

Directional antennas are antenna who forms its beams in a particular direction. There are two main kinds of directional antennas, single beam antennas and multibeam antennas [13]. In single beam antenna, there is only one beam active at a given time because of only one transceiver. Figure 1 shows the single beam antenna. Due single transceiver, multiple transmission/reception is not possible. On the other hand, the multibeam directional antenna can forms multiple beams in any direction. Figure 2.shows the multibeam directional antenna. In the figure we can see that, there is multiple transceiver forms multiple beams. Therefore, simultaneous transmissions or receptions are possible. However, it is not possible that at a same time some beams transmit data and others receive data [14].

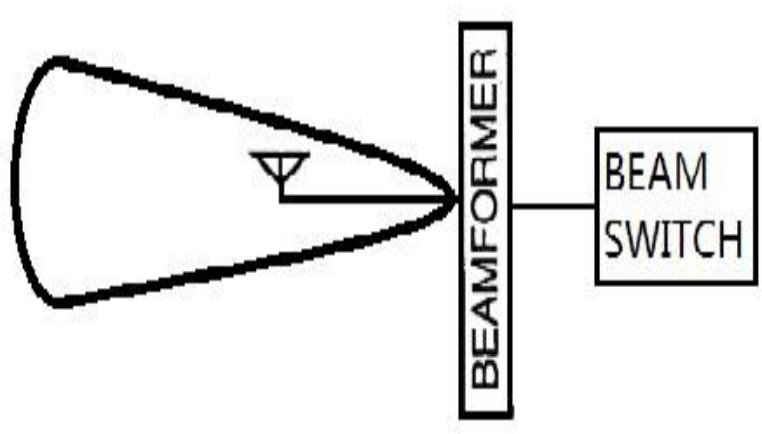

Figure 1.Single Beam Directional Antenna

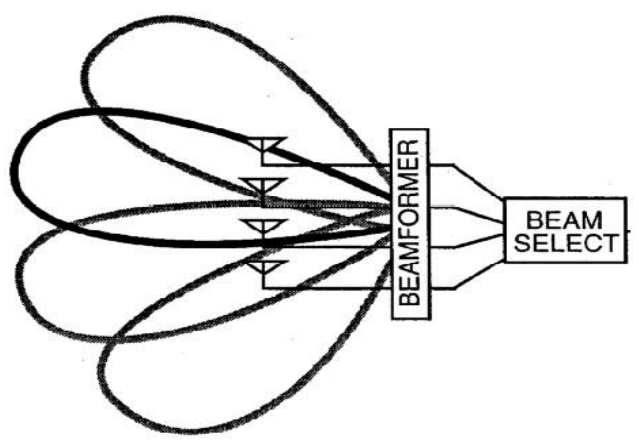

Figure 2.Multibeam Directional Antenna

\section{Directional MAC protocols}

\section{i. $\quad$ Directional MAC1 (DMAC1)}

Ko. YB et al [6] proposed" MAC protocol with directional antennas for deafness avoidance in Ad Hoc network". They first efforts to adapt the IEEE 802.11MAC DCF scheme for directional antennas. Its key feature is the usage of directional RTS frame. On one hand, it narrows the area in which an unintended receiver can overhear the RTS frame and thus significantly relieves the exposed terminal problem. On the other hand, by recording the directions from which the CTS frames are recently overheard and then blocking the antenna elements in the corresponding sectors. A node is further allowed to transmit in the directions that will not collide with other data transmissions, In addition to relieving the hidden terminal problem. The big issue with directional MAC is that, finding the intending receiver location to initiate communication. This paper also suffers with this problem. The authors took GPS support for neighbour location.

\section{ii. $\quad$ Directional MAC2 (DMAC2)}

Nasipuri et al [7] proposed" A MAC protocol for mobile ad hoc networks using directional antennas." In this paper they exploits the ability of the receiver to determine the direction of the arriving frame in order for the transmitting and the receiving nodes to learn each other's direction. In this scheme, RTS and CTS packets are omnidirectional, whereas DATA and ACK packets are directional.

In contracts to directional MAC1, it accomplishes location tracking in an on-demand manner, instead of in a pro-active manner. However, since it uses omnidirectional antennas to transmit both the RTS and CTS frames, it does not have the benefits of using directional RTS frame as in directional MAC1.

iii. $\quad$ Directional MAC3 (DMAC3) 
Gossain et al. [8] propose "Cross-layer directional antenna MAC protocol for wireless ad hoc networks". In this paper they uses multibeam antenna. The nodes send a "hello packet" to their one hop neighbor time to time to know its location. At last the all node of the network knows its neighbor's location. If a node has packet first it does directional RTS/CTS handshake. After successful RTS/CTS handshake, the sender and receiver nodes send RTS and CTS respectively towards their neighbor by using their other beams. The idle nodes sense the channel omnidirectional. It defeats the shortcoming of previous MACs by sending the optimized circular RTS/CTS after the successful RTS/CTS handshake of sender and receiver. However, the omnidirectional reception generates asymmetry in gain in the network which increases the hidden node problem.

\section{iv. Directional MAC4 (DMAC4)}

Alam et al. [9] proposed "Neighbor initiated approach for avoiding deaf and hidden node problems in directional MAC protocol for ad-hoc networks". In this paper they claim that, their scheme overcomes the deaf and hidden node problems. If a node has packet, it transmits RTS to its neighbors simultaneously through its multibeam antenna. The receiver replies with CTS through its multibeam antenna. After successful RTS/CTS they start data communication through the beams pointed towards each other (sender/receiver). The other beams of the communicating nodes are blocks until communication going on. Since, the directional antenna has big feature is spatial reuse. Therefore, to increase the spatial reuse they proposed the transmission of Neighbor Information Packet (NIP). The purpose of transmitting the packet is to inform those nodes who just finish their communication about other ongoing communication in the network.

\section{Comparison of MAC Protocols}

In this section we are going to comparison the MAC protocols, which are described above. They are IEEE 802.11, MACA and MACAW, FAMA, DMAC1, DMAC2, DMAC3 and DMAC4.

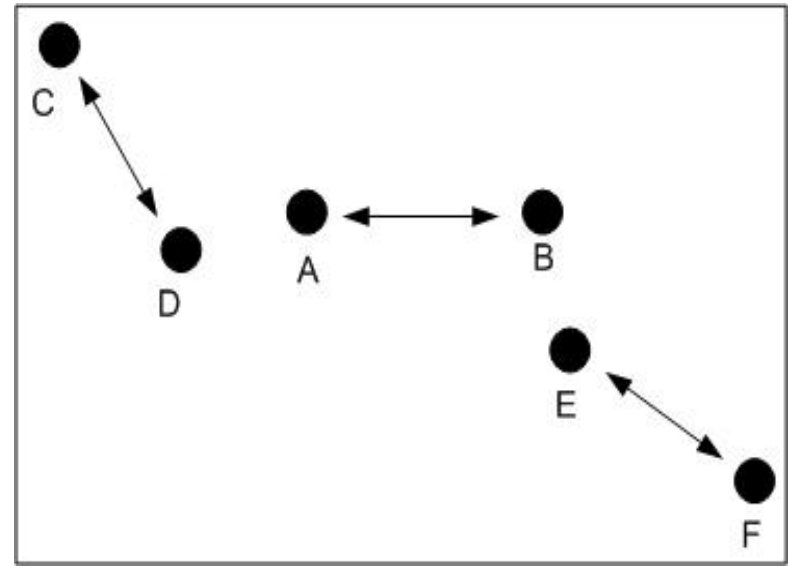

Figure 3. Spatial Reuse Example

The spatial reuse in the ad hoc networks expands effective network capacity and improves its performance. We use the following example, as shown in Figure 3, to analyze the different capabilities in spatial reuse among the MAC protocols in Table I. Node A-B, node $\mathrm{C}-\mathrm{D}$ and node $\mathrm{E}-\mathrm{F}$ can communicate at same time in the same network. For omnidirectional MAC protocols, the node AB, CD and EF should not be within the range of each other. For directional MAC protocols, the direction of the communication of those node pair should not be same. Therefore, the directional MAC protocols increases the spatial reuse, which expands the network capacity and improves the overall throughput in the network.

In the table 1, OMNI stands for omnidirectional antenna, DIRdirectional antenna, BEB means Binary Exponential Backoff and the MILD is Multiple Increase Linear Decrease backoff.

TABLE 1. Features of MAC Protocols

\begin{tabular}{|c|c|c|c|c|c|c|c|c|}
\hline $\begin{array}{l}\text { Protocol } \\
\text { name }\end{array}$ & $\begin{array}{l}\text { Antenna } \\
\text { Type }\end{array}$ & $\begin{array}{l}\text { Carrier } \\
\text { Sensing }\end{array}$ & $\begin{array}{l}\text { Virtual } \\
\text { Sensing }\end{array}$ & $\begin{array}{l}\text { With } \\
\text { ACK }\end{array}$ & $\begin{array}{l}\text { Backoff } \\
\text { Algorithm }\end{array}$ & $\begin{array}{l}\text { Hidden } \\
\text { Terminal free }\end{array}$ & $\begin{array}{l}\text { Exposed Terminal } \\
\text { free }\end{array}$ & $\begin{array}{l}\text { Basic } \\
\text { Protocol }\end{array}$ \\
\hline IEEE 802.11 & OMNI & YES & YES/NO & BEB & YES/NO & $\mathrm{NO}$ & $\mathrm{NO}$ & - --------- \\
\hline MACA & OMNI & $\mathrm{NO}$ & YES & $\mathrm{NO}$ & BEB & YES & YES & ------------ \\
\hline MACAW & OMNI & NO & YES & YES & MILD & YES & YES & MACA \\
\hline FAMA & OMNI & YES/NO & YES & NO & BEB & YES & YES & MACA \\
\hline DMAC1 & DIR & YES & YES/NO & YES & BEB & YES/NO & YES & IEEE 802.11 \\
\hline DMAC2 & DIR & YES & YES/NO & NO & BEB & YES/NO & YES/NO & IEEE 802.11 \\
\hline DMAC3 & DIR & YES & YES/NO & YES & BEB & YES/NO & YES/NO & IEEE 802.11 \\
\hline DMAC4 & DIR & YES & YES/NO & YES & $\overline{B E B}$ & NO & $\mathrm{NO}$ & IEEE 802.11 \\
\hline
\end{tabular}




\section{Performance Evaluation}

In this section we discuss about our simulation model and performance of the MAC protocols.

\section{A. Simulation Model}

We used OPNET 14.5, for simulate the MAC protocols. This simulation tool provides an antenna pattern editor. Using this antenna pattern editor we have designed antenna for directional MAC protocol, which is depicted in Figure . The omnidirectional MAC protocols uses default isotropic antenna pattern provided by OPNET 14.5.

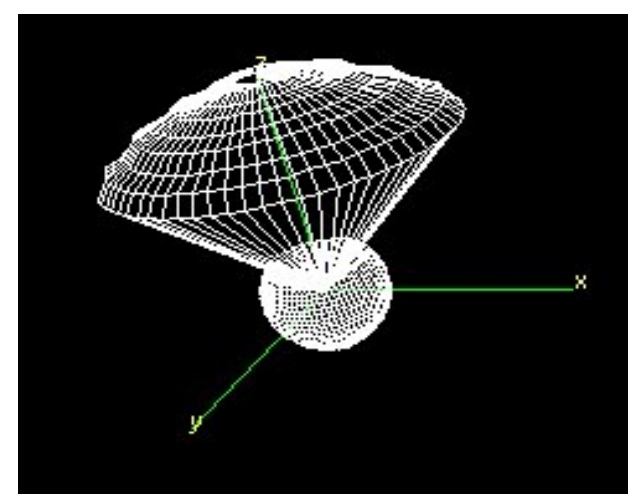

Figure 4. Antenna Model

We modified used default node model for omnidirectional MAC protocols, provided by the simulator. The node model is depicted in figure 5. In the figure source generates packet exponentially, Wlan_MAC_intf give the environment of upper layers to MAC layer. Wireless_lan_MAC manages the MAC layer. In this processor, the MAC process model runs. We modified the process model as per the MAC protocols require.

We modified the omnidirectional node for directional MAC protocols as Figure 6. The node model is similar as omnidirectional node model, only difference between them is that, in directional node model we added directional antenna whereas, in the omnidirectional node model we uses default isotropic antenna.

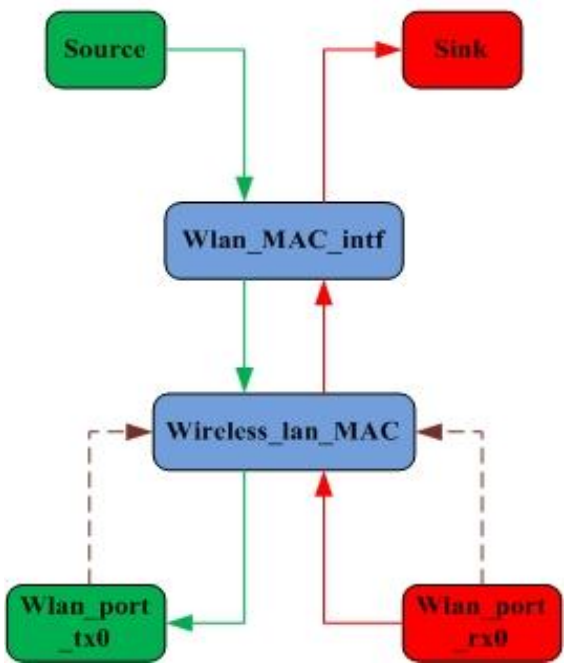

Figure 5.Node Model for Omnidirectional MAC

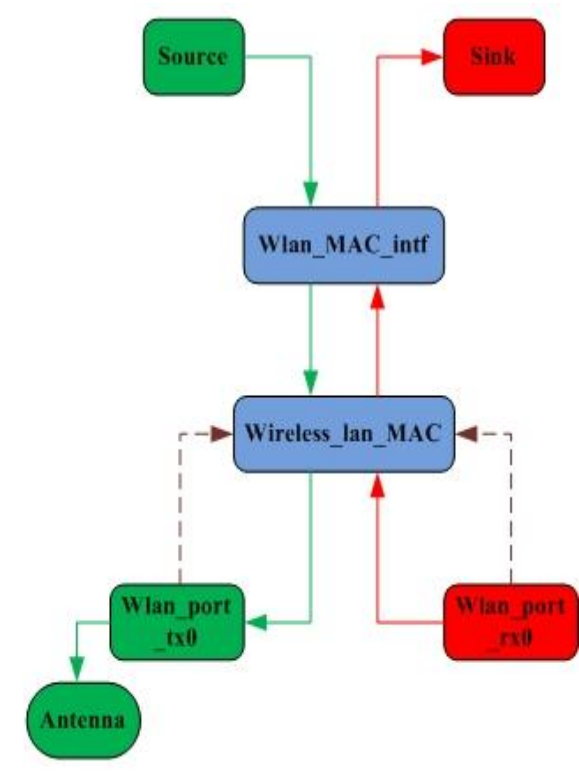

Figure 6. Node Model for Directional MAC

In our simulation we designed a scenario with sixteen nodes which is randomely distributed in 500 X 500 meters. Figure 7 shows the senario and table 2 contains the simulation parameter.

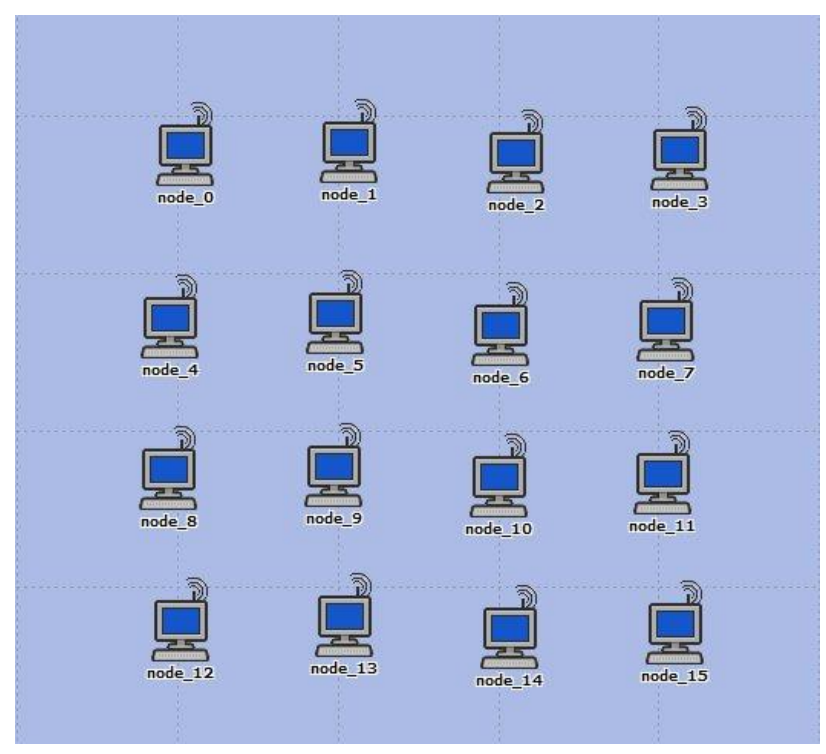

Figure 7.Simulation Scenario

Each simulation run for 600 seconds with 128 different seeds. We average the results getting by 10 simulation runs.

\section{B. Simulation Results}

In this section we evaluate the simulation results in terms of aggregate throughput and delay. The throughput is defined as the average rate of successful message delivery over a communication channel.

Figure 8.Shows the throughput versus traffic load performance results of the omnidirectional and directional MAC protocols. In the figure we can see that the DMAC4 and DMAC3 performed better among the MAC protocols. The reason of the better performance of those directional MAC is getting better spatial reuse of the network and successfully overcome the hidden and deaf node problem. Among the above MAC, the DMAC4 performance is better because there is no hidden node problem due to asymmetry in gain, whereas the DMAC3 suffers with this 
problem. The DMAC1 and DMAC2 does not perform as DMAC3 and DMAC4, because they suffers from hidden and deaf node problem. Although in directional MAC, spatial reuse is better than the omnidirectional MAC protocols, but due to hidden and deaf node problem most of the packet collided in DMAC1 and DMAC2.

Table2. Simulation Parameter

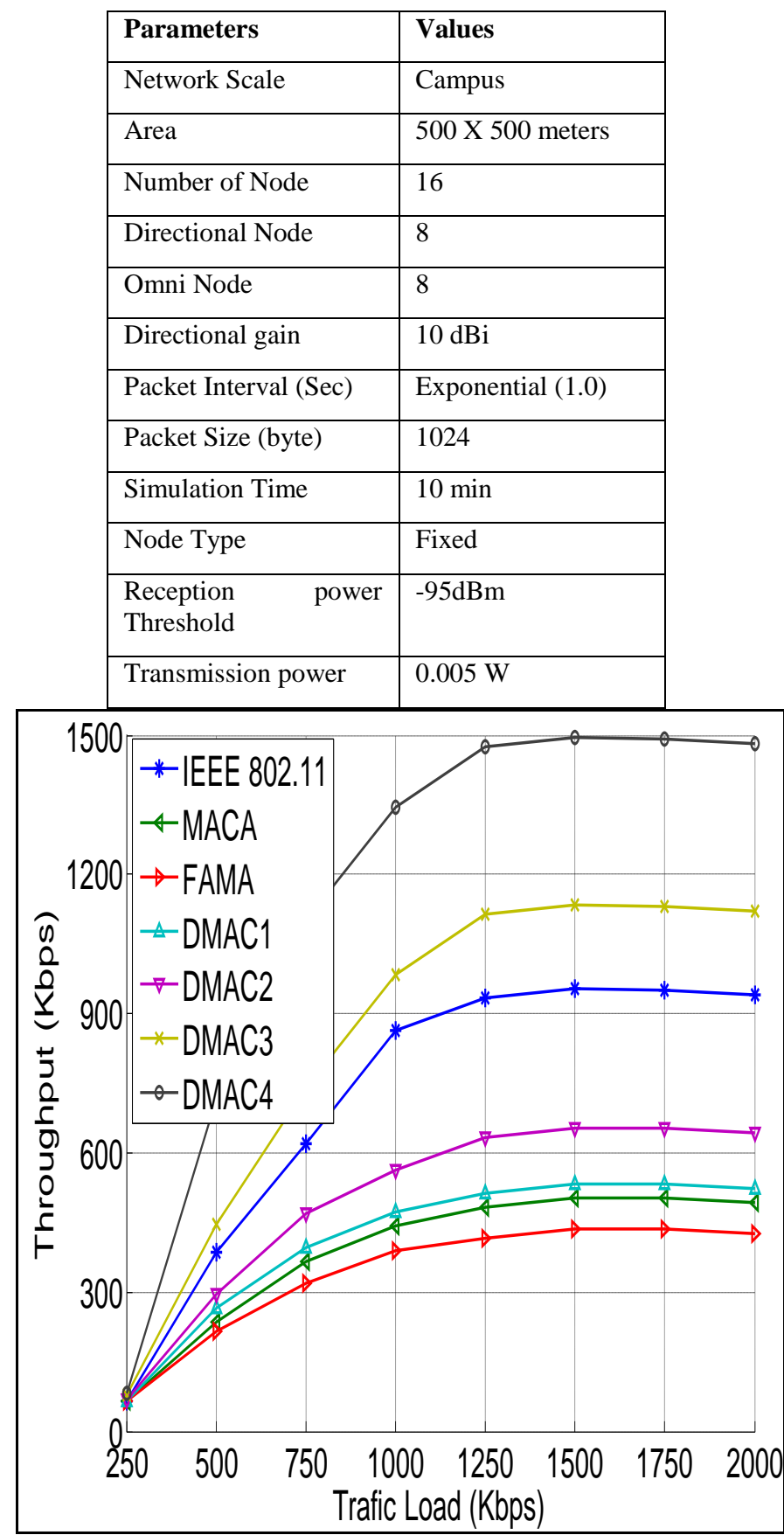

Figure 8. Throughput of the MAC protocols

Among omnidirectional MAC protocols, IEEE 802.11 performance is better than others. The IEEE 802.11 performance is also better than the DMAC1 and DMAC2. The reason of the better performance of the MAC is less hidden node and deaf node problem as well as no exposed node problem, whereas the other omnidirectional MAC protocols suffers from those problems.

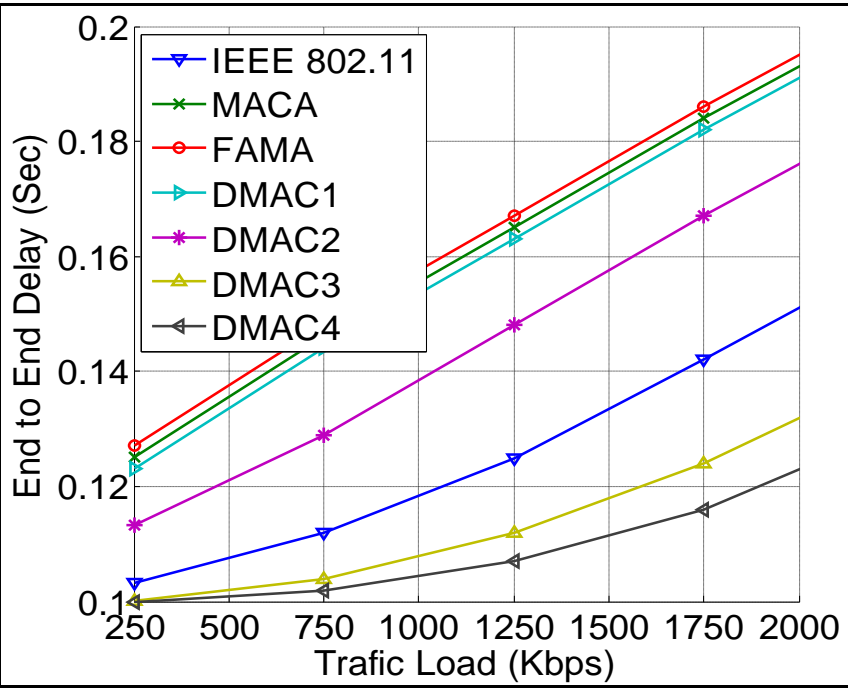

Figure 9. End to End Delay of the MAC protocols

Figure 9 shows the End to End Delay versus traffic load of the MAC protocols. In the figure, we can see that the omni directional MAC protocols except IEEE 802.11 and the DMAC1 and DMAC2 have more delay than others. The delay of DMAC3 and DMAC4 has less than IEEE 802.11. The DMAC4 delay is lesser than the DMAC3, because there is no double transmission of RTS/CTS before data communication. The IEEE 802.11 delay is more than the DMAC3 and DMAC4 but lesser than the others. The reason is less collision of the packet in the network, whereas the other MAC protocols (except DMAC3 and DMAC4) suffer from the hidden and deaf node problem.

\section{CONCLUSIONS}

We have completed a relative study of the omnidirectional and directional MAC protocols for ad hoc networks. Our comparison found that the directional MAC protocols achieves better spatial reuse than omnidirectional MAC protocols. Some directional MAC is highly suffers from hidden and deaf node problem, so they couldn't performed well. Among omnidirectional MAC protocols IEEE 802.11 performance is better than others i.e. it overcomes the exposed node problems, whereas the other omnidirectional MAC protocols suffers from these problems. Overall, we can say that to achieve better performance we need to improve the Directional MAC protocols.

\section{ACKNOWLEDGMENT}

This work was supported by college of e-Learning \& Distance Learning Deanship, King Saud University, Riyadh, Saudi Arabia.

\section{REFERENCES}

[1] Kleinrock, L.; Tobagi, F.; "Packet Switching in Radio Channels: Part I--Carrier Sense Multiple-Access Modes and Their Throughput- Delay Characteristics," Communications, IEEE Transactions on , vol.23, no.12, 1400- 1416, Dec 1975 doi:10.1109/TCOM.1975.1092768

[2] Phil Karn. MACA a new channel access method for packet radio. In Computer Networking Conference, volume 9th, pages $134-140,1990$

[3] Vaduvur Bharghavan, Alan Demers, Scott Shenker, and Lixia Zhang. MACAW: A media access protocol for wireless LAN's. In Proceedings of the SIGCOMM '94 Conference on Communications Architectures, Protocols and Applications, pages 212-225, August 1994. 
[4] Chane L. Fullmer and J. J. Garcia-Luna-Aceves, "Floor Acquisition Multiple Access (FAMA) for Packet-Radio Networks," in ACMSIGCOMM,Cambridge, MA, Aug. 2Wep. I 1995, 262-73.

[5] IEEE 802.11, "Part 11: Wireless LAN Medium Access Control (MAC) and Physical Layer PHY) Specifications", IEEE 802.11 Std, Aug 1999.

[6] Young-Bae Ko, Vinaychandra Shankarkumar, and Nitin H. Vaidya, "Medium Access Control Protocols Using Directional Antennas in d Hoc Networks," in IEEE INFOCOM, Tel Aviv, Israel, Mar. 26-30 2000, pp. 13-21.

[7] Nasipuri, A., Ye, S., You, J., \& Hiromoto, R. E. (2000). A MAC protocol for mobile ad hoc networks using directional antennas. In IEEE wireless communications and networking conference (WCNC 2000) (pp. 1214-1219). doi:10.1109/WCNC.2000. 904804

[8] Gossain, H., Cordeiro, C., Joshi, T., \& Agrawal, D. P. (2006). Cross-layer directional antenna MAC protocol for wireless ad hoc networks: Research Articles. Wireless Communications \& Mobile Computing, 6(2), 171-182. doi:10.1002/wcm.v6:2
[9] Alam, Md. Nasre. Hussain, Md. Asdaque. Kwak, Kyung Sup "Neighbor initiated approach for avoiding deaf and hidden node problems in directional MAC protocol for ad-hoc networks" Wireless Networks, October 2012, DOI: 10.1007/s11276-012-0510-8.

[10] Radio/Wireless Models, OPNET, http:/www.opnet.com/, 2010.

[11] [1] Z. Huang and C.-C. Shen, "A comparison study of omnidirectional and directional MAC protocols for ad hoc networks," Global Telecommunications Conference, 2002. GLOBECOM '02. IEEE, vol. 1. pp. 57-61 vol.1, 2002.

[12] L. Kleinrock and M.O. Scholl, "Packet Switching in Radio Channels: New Conflict - Free Multiple Access Schemes," IEEE Trans. Commun., vol. Com - 28, 1015-29, 1980.

[13] J.H. winters, Smart antenna techniques and their application to wireless Ad-hoc networks, IEEE Wireless Communications 13 (4) (2006) 77-83.

[14] Lehne, P.H., Pettersen, M.: An overview of smart antenna technology for mobile communications systems. IEEE Communications Surveys, Vol.2, No.4, (1999) 2-13. 